\title{
Dental emergencies presenting to maxillofacial units during the COVID-19 pandemic: a five-centre UK hospital study
}

\author{
Kristian K. Blackhall ${ }^{\star 1}$ and Rabindra P. Singh ${ }^{2}$
}

\section{Key points}

Access to emergency dental care was unpredictable and significantly limited, particularly during the beginning of the COVID

19 pandemic period.
A proportion of patients with dental emergencies attended hospital emergency services with nowhere else to turn, often with more severe symptoms than normally encountered, having had no treatment and often multiple antibiotic courses.
Treatment of patients in a dental setting during the pandemic period was challenging due to transmission risk factors and aerosol generation.

\begin{abstract}
Introduction Coronavirus disease (COVID-19) represents an unprecedented challenge to healthcare. Provision of dental services was significantly affected, with limited provision for emergencies resulting in patients increasingly attending hospitals

Objectives and methods The aim of the study was to assess the provision of emergency dental services by the maxillofacial departments during the initial COVID-19 pandemic lockdown in the United Kingdom. At the outset of the first lockdown, we initiated a prospective study to monitor the presentation and management of dental emergencies at five hospital trusts. Data were collected onto an online live database until the lockdown relaxation.

Results Of a total of 211 dental emergencies, 156 were infection-related, 42 were trauma-related and 12 were cases of post-operative complications. A proportion of patients (76\%) could have been treated in primary care, with $52 \%$ of presentations having already been 'triaged' on several occasions by urgent dental hubs and given multiple antibiotic courses, rather than any direct treatment.
\end{abstract}

Conclusion There is a need to restructure emergency dental service provision as well as planning for possible further COVID-19 spikes and future pandemics.

\section{Introduction}

A coronavirus disease (COVID-19) outbreak originated in Southern China in late 2019 and spread to multiple countries worldwide. ${ }^{1,2}$ On 29 January 2020, the first case of COVID19 was diagnosed in the UK. ${ }^{3}$ The virus has been shown to spread by person-to-person transmission ${ }^{4,5}$ and is widely accepted to travel via an airborne vector. The virus can be present on aerodigestive mucosal surfaces and in saliva, and can cause potentially dangerous

'Specialty Doctor in Oral and Maxillofacial Surgery, Department of Oral and Maxillofacial Surgery, Salisbury District Hospital, Salisbury, Wiltshire, SP2 8BJ, UK:

${ }^{2}$ Consultant in Oral and Maxillofacial Surgery, Department of Oral and Maxillofacial Surgery, Southampton University

Hospital, Tremona Road, Southampton, S016 6YD, UK.

*Correspondence to: Kristian Blackhall

Email address: kristian.blackhall@nhs.net

Refereed Paper.

Accepted 9 November 2020

https://doi.org/10.1038/s41415-020-2499-1 respiratory symptoms and complications. ${ }^{6,7}$

The initial lockdown measures were effected in the UK on 24 March 2020, shortly following the declaration of a global pandemic by the World Health Organisation. ${ }^{8,9}$ Among the guidelines issued, the Chief Dental Officer stated that routine dental patients should be cancelled, non-emergency treatment deferred and, if possible, any emergency dental care be regionally consolidated. ${ }^{10,11} \mathrm{Key}$ to the dental guidelines was the avoidance of all aerosol generating procedures (AGPs), which effectively prevented a proportion of routine dental treatments.

On the second day of the first national lockdown, we initiated a study to monitor the presentation and management of dental emergencies at five major National Health Service (NHS) hospital trusts. The aims of this study were to analyse dental emergency patient presentations, their management and to provide discussion from an acute maxillofacial hospital perspective on activity during the first pandemic lockdown period, as well as our strategies employed to adapt and maintain service provision.

\section{Materials and methods}

This study involved data collection from multiple acute NHS hospital trusts across the south west region of the UK: University Hospital Southampton, St Richard's Hospital (Chichester), Salisbury Hospital, Poole Hospital and Queen Alexandra Hospital (Portsmouth). The population catchment of these hospitals is approximately 4 million, which is equivalent to $6 \%$ of the total UK population. ${ }^{12}$ The data for this study were collected prospectively for a six-week period from the start of the first lockdown to the easing of the tight lockdown restrictions. This study was registered within the respective hospitals' clinical governance team as a service evaluation study. 
Table 1 Patient data related to dental emergency consultations

\begin{tabular}{l|l} 
Variables & $\begin{array}{l}\text { Number of patients (\%) } \\
\text { (Total number of patients = 211) }\end{array}$ \\
\hline Age & $\begin{array}{l}\text { Mean age: } 38 \\
\text { Range: 0-95 }\end{array}$ \\
\hline Gender & \multicolumn{2}{|l}{} \\
\hline Male & $115(54.5)$ \\
\hline Female & $96(45.5)$ \\
\hline
\end{tabular}

\begin{tabular}{l|l}
\hline Source of referral & $137(64.9)$ \\
\hline Hospital A\&E & $31(14.7)$ \\
\hline Dentist & $13(6.2)$ \\
\hline GP & $30(14.2)$ \\
\hline Other (walk-in centre/emergency from other specialty (eg ENT) & \\
\hline Care setting & $36(17.1)$ \\
\hline Inpatient & $175(82.9)$ \\
\hline Outpatient & \\
\hline Treatment modality & $127(60.2)$ \\
\hline Local anaesthetic & $18(8.5)$ \\
\hline General anaesthetic & $66(31.3)$ \\
\hline Conservative management &
\end{tabular}

\begin{tabular}{l|l}
\hline Grade of consulting/treating clinician & $17(8.1)$ \\
\hline Consultant & $78(37.0)$ \\
\hline Middle grade & $116(54.9)$ \\
\hline Dental core/foundation trainee &
\end{tabular}

\begin{tabular}{|c|c|}
\hline \multicolumn{2}{|c|}{ Patient's COVID-19 status } \\
\hline Unknown & $203(96.2)$ \\
\hline Positive (tested) & $0(0)$ \\
\hline Suspected positive & $3(1.4)$ \\
\hline Negative (tested) & $5(2.4)$ \\
\hline \multicolumn{2}{|c|}{$\begin{array}{l}\text { Could have been treated in primary care } \\
\text { (ie not necessitating specialist maxillofacial-trained clinicians to treat) }\end{array}$} \\
\hline Yes & $160(75.8)$ \\
\hline No & $51(24.2)$ \\
\hline
\end{tabular}

This study involved capturing data prospectively using a custom-designed smartphone app or web form to allow for easy and efficient data entry. Data were centrally stored in an online 'live' whiteboard where they were automatically processed by several algorithms, allowing for real-time presentation of the results. These data were continually accessible to the collaborators and hence allowed any issues surrounding patient care, referrals and emergency management to be picked up in a timely manner, and changes of pathways and practices implemented as a short timeframe by the lead author of this paper, who holds an interest in computer programming and algorithmic mathematical programming languages alongside their clinical work in the maxillofacial unit.
Data inclusion criteria involved any patients presenting with acute dental emergencies via any route: accident and emergency (A\&E); walk-ins and out-of-hours; urgent dental care (UDC) hub referrals; or primary care referrals. All dental emergencies including dental infections, dental trauma and any other cause were included. Specific data points measured and captured are displayed in Table 1. No patient-identifiable information was recorded. Access to the application and online database was encrypted and restricted to the participants in the maxillofacial units. Any record entered that did not contain all the data points was automatically flagged by the system and held in isolation from the main database until such time as the missing data were added.

\section{Results}

There were a total of 211 dental emergencies which presented throughout the five hospitals. One hundred and forty-five patients received direct intervention and 66 patients were treated with advice and more conservative management strategies. The detailed data on a range of parameters examined are listed in Table 1.

There were 156 infection-related cases, 42 trauma-related cases and 12 cases of postoperative complications. The vast majority of patients who attended the hospitals were treated as outpatients $(82.9 \%, \mathrm{n}=175)$, with 127 receiving treatment under local anaesthesia. A small proportion of patients required a higher level of care and admission to the hospital $(\mathrm{n}=36)$, with 18 ultimately having emergency treatment under general anaesthesia. Over two-thirds (72\%) of patients presenting with a dental emergency had attempted to seek treatment from their own dentist or a UDC hub via the NHS 111 service, but were either declined treatment or were unable to secure an appointment. Over half (52\%) of patients who presented with emergencies had already been 'triaged' or seen on several occasions by their local dentist or UDC and were simply provided with one or several concurrent courses of oral antibiotics.

\section{Discussion}

During the data collection period, the use of an online platform (which fed through both a custom-programmed smartphone app and a web app) allowed for an excellent 
and consistent uptake, with the results pooled centrally and accessible to all collaborators. This allowed for data analysis and gave valuable insights into patient management as well as staff timetable management during the peak of the pandemic.

Dental emergencies comprise a significant proportion of hospital A\&E attendances, ${ }^{13}$ and generate a steady referral source for secondary and tertiary care providers such as oral and maxillofacial surgery (OMFS). ${ }^{14,15}$ Patients presenting to A\&E departments consistently and regularly report having nowhere else to turn to with their acute dental problem, ${ }^{13,15}$ or simply having (sometimes multiple) antibiotic courses provided rather than any definitive treatment. This has become much more significant and burdensome on the hospital system and specialties such as OMFS of late due to the pandemic lockdown, with the closure of much of the primary dental care sector and emergency dental care being restricted to a handful of urgent dental hubs.

With the closure of primary dental care services and the consolidation of UDC into regional hubs, patients with an acute dental issue were instructed to contact their own dentist who would triage them virtually. If deemed sufficiently in severe pain or categorised as a higher-level dental emergency, they are then referred by their dentist to the nearest UDC hub. For nonurgent cases, dentists were guided to use the ' 3 As' - advice, antimicrobials and analgesia - when managing patients with dental problems. ${ }^{16}$

In our interactions with patients who attended the hospital, we repeatedly received comments and complaints about the lack of provision of emergency dental care and the difficulty in accessing it. Indeed, during the pandemic period, the availability was severely limited. ${ }^{17}$ Furthermore, the UDC hubs had a limited capacity to treat patients as they required adequate level 3 personal protective equipment (PPE) due to the significant risks if aerosol generation was planned. Limited access was also due to the need to deep-clean between patients and appropriate downtime after aerosol generation to reduce viral transmission risks.

Dentistry, alongside OMFS and ear, nose and throat (ENT) specialties, is particularly at a high risk during the pandemic period due to procedures which may lead to aerosol generation. ${ }^{18,19}$ It has been shown that, in procedures such as surgical bone removal, cavity preparation and root canal access during which aerosol is generated through using high-speed handpiece and ultrasonic devices, these particles can be up to 50 micrometres in size, hence presenting an infection risk if loaded with pathogens. ${ }^{19}$ This highlights a significant risk of spread of infectious agent. High-speed dental handpieces in particular generate significant aerosol, and it has long been considered an occupational hazard for dentists and practitioners that regularly use these instruments. ${ }^{20}$ It has even been shown that these aerosols can spread up to $1.5 \mathrm{~m}$ from the operator into the environment. ${ }^{21}$

Increased demand, capacity limitations, possible aerosol generation, need for stringent PPE usage and deep-cleaning resulted in a throttled emergency dental service that could not necessarily meet the demands placed upon it. The resultant influx of dental patients to acute hospitals and $A \& E$ is therefore not entirely unexpected. Additionally, based on case-by-case analysis of the nature of dental abscess encountered, the clinicians participating in the study felt the number of more significant and well-established dental abscesses presenting had increased due to several patients being on extended-duration antibiotic courses, with limited access to definitive treatment such as tooth extraction or endodontic procedures. Regional UDC hubs were set up but were gradual, and the information about them was scarce and may have been confusing for primary dental care providers. Clear regional patient pathways would have helped all stakeholders during the first pandemic lockdown.

In preparation for the perceived need for patient care and increasing referrals, several of our OMFS units developed their own patient access and triage systems. Surgery rooms were set aside and designated as treatment areas for acute dental patients, allowing for appropriate use of PPE, rest periods after AGPs and deep cleaning. This allowed for some provision of direct patient care, although was mainly limited to tooth extractions, incision and drainage of abscesses or splinting of traumatised teeth. Avoiding the use of powered surgical instruments such as drills and handpieces was recommended and hence the more senior (ie middle grades and above) members of the team usually undertook most procedures, in order to reduce the risk of tooth fracture and progression to surgical removal. Senior staff undertaking more procedures also meant that intervention was more efficient due to their clinical judgement and experience, which also allowed for some of the junior staff to be relatively free for redeployment purposes.

An increasing presentation of wellestablished dental abscesses was noted; patients having had multiple courses of oral antibiotics, there was plenty of time for abscesses to form defined collections within the associated facial spaces. Provision of antibiotics alone is not an ultimate curative solution to acute dental infection; ${ }^{22}$ only definitive treatment such as tooth extirpation or extraction can ensure the infective process is halted and the cause is removed. ${ }^{23}$ There is an urgent need to invest further in the provision of emergency dental care and increase the number of hubs available in which patients could receive the necessary intervention for their acute condition.

\section{Conclusion}

Having seen a significant change in the provision for emergency dental care during the first lockdown of the COVID-19 pandemic, there is a clear need to plan regional UDC hubs and workforce accordingly for further possible peaks of the COVID-19 disease and future pandemics. Increased direct patient care is required to alleviate and halt acute dental problems. Dental procedures such as tooth extirpation or extraction rather than prescription of antibiotics and analgesics are highly indicated, and are backed by the evidence demonstrating its key effectiveness and that it is the principal method of treating dental emergencies.

The increasing burden of dental emergencies presenting to hospital A\&E departments as well as to specialties such as OMFS should be anticipated and planned for during a lockdown period. Provision and adaptation of service structure should be implemented to accommodate the increasing number and severity of dental infections.

\section{Conflict of interest}

We have no conflicts of interest.

\section{Acknowledgements}

We would like to express our gratitude to all core and foundation trainees, speciality registrars and consultants at the Health Education Wessex who supported and helped to make this study possible. Institutional approval was obtained for the study. Patient consent was not required. 
who-announces-covid-19-outbreak-a-pandemic (accessed November 2020).

\section{References}

1. Zhu N, Zhang D, Wang W et al. A Novel Coronavirus from Patients with Pneumonia in China, 2019. N Engl J Med 2020; 382: 727-733.

2. Wang C, Horby P W, Hayden F G et al. A nove coronavirus outbreak of global health concern. Lancet 2020; 395: 470-473.

3. Moss P, Barlow G, Easom N et al. Lessons for managing high-consequence infections from first COVID-19 cases in the UK. Lancet 2020; DOI: 10.1016/ S0140-6736(20)30463-3.

4. Liu T, Hu J, Kang M et al. Transmission dynamics of 2019 novel coronavirus (2019-nCoV). 2020. Available online at http://dx.doi.org/10.2139/ssrn.3526307 (accessed November 2020)

5. Chan J F-W, Yuan S, Kok K-H et al. A familial cluster of pneumonia associated with the 2019 novel coronavirus indicating person-to-person transmission: a study of a family cluster. Lancet 2020; 395: 514-523.

6. Lu C, Liu X, Jia Z. 2019-nCoV transmission through the ocular surface must not be ignored. Lancet 2020; DOI: 10.1016/S0140-6736(20)30313-5.

7. To K K-W, Tsang O T-Y, Chik-Yan Yip C et al. Consistent detection of 2019 novel coronavirus in saliva. Clin Infect Dis 2020; 71: 841-843.

8. World Health Organisation. WHO announces COVID-19 outbreak a pandemic. 2020.

Available at https://www.euro.who.int/ en/health-topics/health-emergencies/ coronavirus-covid-19/news/news/2020/3/
9. UK Government. Coronavirus (COVID-19): what you need to do. 2020. Available online at https://www. gov.uk/coronavirus (accessed May 2020)

10. Royal College of Surgeons of England. COVID-19: Information Hub. 2020. Available at https://www. rcseng.ac.uk/dental-faculties/fds/coronavirus/ (accessed May 2020).

11. The British Association of Dental Nurses. COVID-19 Chief Dental Officer Summary of Guidance. 2020. Available at https://www.badn.org.uk/News/COVID19-Chief-Dental-Officers-summary-of-guidance.aspx (accessed May 2020).

12. Care Quality Commission. Inspection Reports. 2020. Available online at https://www.cqc.org.uk/ what-we-do/how-we-do-our-job/inspection-reports (accessed May 2020).

13. Yap M, Kok M-R, Nanda S et al. Hospital admissions and emergency department presentations for dental conditions indicate access to hospital, rather than poor access to dental health care in the community. Aust J Prim Health 2018; 24: 74

14. Currie C C, Stone S J, Connolly J et al. Dental pain in the medical emergency department: a crosssectional study. J Oral Rehabil 2016; 44: 105-111.

15. Bowden E, Cashman H. Burden to A\&E. Br Dent J 2017; 223: 3-4.

16. NHS England. Urgent dental care guidance and standard operating procedure. 2020. Available online at https://www. england.nhs.uk/coronavirus/publication/ covid-19-guidance-and-standard-operatingprocedure-urgent-dental-care-systems-in-thecontext-of-coronavirus/ (accessed July 2020).

17. Grossman S, Sandhu P, Sproat C, Patel V. Provision of dental services at a single institution in the UK's epicentre during the COVID-19 pandemic. Br Dent J 2020; 228: 964-970.

18. Magennis P, Coulthard P. BAOS \& BAOMS - Guidance for the Care of OMFS and Oral Surgery Patients Where COVID is Prevalent. 2020. Available at https://www.baoms.org.uk/_userfiles/pages/files/ professionals/covid_19/baos_baoms_covid19 postions paper final.pdf (accessed May 2020).

19. Thomas R J. Particle size and pathogenicity in the respiratory tract. Virulence 2013; 4: 847-858.

20. Szymańska J. Dental bioaerosol as an occupationa hazard in a dentist's workplace. Ann Agric Environ Med 2007; 14: 203-207.

21. Marchand G, Duchaine C, Lavoie J et al. Bacteria emitted in ambient air during bronchoscopy-a risk to health care workers? Am J Infect Control 2016; 44: 1634-1638.

22. Tampi M P, Pilcher $L$, Urquhart 0 et al. Antibiotics for the urgent management of symptomatic irreversible pulpitis, symptomatic apical periodontitis, and localized acute apical abscess. J Am Dent Assoc 2019; DOI: 10.1016/j.adaj.2019.09.011.

23. Cope A, Francis N, Wood F, Mann M K, Chestnut I G. Systemic antibiotics for symptomatic apical periodontitis and acute apical abscess in adults. Cochrane Database Syst Rev 2014; DOI: 10.1002/14651858.CD010136.pub2. 